\title{
Editorial
}

Z Gerontol Geriat 2020 · 53:99

https://doi.org/10.1007/s00391-020-01707-4

(c) Springer Medizin Verlag GmbH, ein Teil von Springer Nature 2020

Wir Menschen verbringen etwa ein Drittel unseres Lebens schlafend. Schlaf ist dabei kein Luxus oder überflüssig. Schlaf ist essenziell für Gesundheit, Immunabwehr, Wohlbefinden, Lebensqualität sowie kognitive und physische Leistungsfähigkeit. Jeder, der einmal schlecht geschlafen hat, kennt die unangenehmen Symptome des Schlafmangels.

Der Schlaf verändert sich mit dem Lebensalter. So wie die Haare grau werden, so „ergraut“ auch der Schlaf im Alter. Hier ist es wichtig, die alterstypischen Veränderungen von krankhaften und damit behandlungsbedürftigen Störungen zu unterscheiden, damit die richtigen $\mathrm{Pa}$ tienten behandelt werden können.

Die Symptome eines gestörten Schlafs sind unspezifisch. Solche Symptome finden wir aber bei vielen unserer Patienten. Zudem klagen mehr als die Hälfte der älteren Menschen über einen wie auch immer gestörten Schlaf. Daher ist es wichtig, dass bei der Behandlung älterer Menschen auch der Schlaf thematisiert werden muss. Oft resultiert aus der Behandlung einer Schlafstörung eine erhebliche Verbesserung, funktionell, emotional und kognitiv.

Am Anfang der Abklärung einer Schlafstörung steht ein Assessment. Auch wenn in der Schlafmedizin viele Instrumente zur Abklärung einer Schlafstörung verfügbar sind, so sind die meisten dieser Instrumente für ältere Patienten selten und für geriatrische $\mathrm{Pa}$ tienten praktisch nicht validiert. Welche Instrumente sich für geriatrische Patienten eignen und wo deren Stärken und Schwächen liegen, wird in einem Beitrag dieses Hefts dargestellt. Dabei muss klar sein, dass ein wesentlicher Schritt die

\author{
Helmut Frohnhofen ${ }^{1,2}$ \\ ${ }^{1}$ Alfried Krupp von Bohlen und Halbach Krankenhaus gemeinnützige GmbH, Essen, Deutschland \\ ${ }^{2}$ Fakultät für Gesundheit, Department für Humanmedizin, Universität Witten-Herdecke, Witten, \\ Deutschland
}

\section{Schlaf und Schlafstörungen im höheren Lebensalter}

Weiterentwicklung und Adaptation von Instrumenten sein wird. Hier besteht Forschungsbedarf, damit das Assessment von Schlafstörungen fest in ein umfassendes geriatrisches Assessment integriert werden kann.

Schlafstörungen werden in der International Classification of Sleep Disorders (ICSD) in der nun verfügbaren 3 . Version in 6 große Gruppen eingeteilt. Die erst Gruppe umfasst die Insomnien. Insomnien sind auch im höheren Lebensalter die häufigste Form einer Schlafstörung. Zwei Beiträge beschäftigen sich mit der Insomnie im höheren Alter. Einmal wird die wechselseitige Beziehung zwischen Insomnien und Komorbiditäten aufgezeigt. In einem zweiten Artikel werden die aktuellen Behandlungsmöglichkeiten der Insomnie vorgestellt und konkreter das verhaltenstherapeutische Verfahren der Stimuluskontrolle erklärt.

$\mathrm{Zu}$ einer weiteren, eigenständigen Gruppe fasst die ICSD die sog. Parasomnien zusammen. Diese weit verbreiteten Phänomen werden oft nicht wahrgenommen oder verkannt, und eine adäquate Behandlung unterbleibt dann. Den häufigsten Parasomnien, deren Abklärung und Behandlung, ist ein eigner Beitrag gewidmet.

Ich wünsche Ihnen viel Freude beim Lesen.

Helmut Frohnhofen

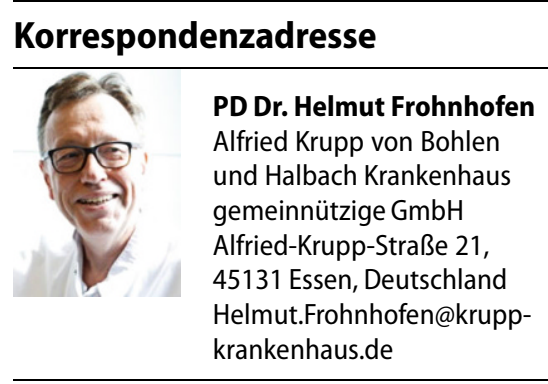

Interessenkonflikt. H. Frohnhofen weist auffolgende Beziehungen hin: Vortragshonorare von Amgen, Heel und Pfizer. 